\title{
Assessment of Cortical Visual Field Representations with Multifocal VEPs in Control Subjects, Patients with Albinism, and Female Carriers of Ocular Albinism
}

\author{
Michael B. Hoffmann, ${ }^{1}$ Birgit Lorenz, ${ }^{2}$ Markus Preising, ${ }^{2}$ and Petra S. Seufert ${ }^{1,3}$
}

Purpose. In human albinism, part of the temporal retina projects abnormally to the contralateral hemisphere. This study was undertaken to test whether this abnormality can be identified with multifocal visual evoked potentials (mfVEPs) and whether it is evident in carriers of ocular albinism (OA1).

Methods. In 12 control subjects, 11 patients with albinism, and 5 female carriers of OA1 monocular pattern-reversal mfVEPs were recorded for 60 locations comprising a visual field of $44^{\circ}$ diameter (VERIS ver. 4.8; EDI, San Mateo, CA). For each eye and each stimulus location interhemispheric difference potentials were calculated and correlated with each other to assess the lateralization of the responses: positive and negative correlations indicate lateralization on same or opposite hemispheres, respectively. Misrouted optic nerves are expected to yield negative interocular correlations. Visual field locations without recordable responses were excluded from the analysis using a signal-to-noise threshold. The analysis also allowed assessment of the sensitivity and specificity of the detection of projection abnormalities.

Results. Sizable mfVEPs were obtained in all control subjects, carriers, and the three patients with albinism who had negligible nystagmus and visual acuity $>0.25$. Ninety-seven percent and $99 \%$ of the visual field locations were identified as normal in control subjects and carriers, respectively. While this indicates a specificity of the procedure of $97 \%$, the sensitivity was estimated as $75 \%$. Finally, in albinism, 55\% percent of the responses were abnormally represented.

Conclusions. In the absence of nystagmus mfVEPs are a powerful tool to identify, in a spatially resolved manner, abnormal visual field representations. No local representation abnormalities were evident in the female carriers of OA1. (Invest Ophthalmol Vis Sci. 2006;47:3195-3201) DOI:10.1167/iovs.051471

$I^{1}$ $\mathrm{n}$ humans, the nasal retina projects to the contralateral hemisphere, whereas the temporal retina projects ipsilaterally. Consequently, the line of decussation that divides crossed from uncrossed fibers normally coincides with the vertical meridian through the fovea. This normal projection of visual fibers from

From the ${ }^{1}$ Visual Processing Lab, Universitäts-Augenklinik, Magdeburg, Germany; ${ }^{2}$ Abteilung fur Kinderophthalmologie, Strabismologie und Ophthalmogenetik, Klinikum der Universität, Regensburg, Germany; and ${ }^{3}$ Sektion für funktionelle Sehforschung, Universitäts-Augenklinik, Freiburg, Germany.

Supported by German Research Council Grant DFG HO-2002/4-1. Submitted for publication November 17, 2005; revised December 23, 2005, and March 2, 2006; accepted May 2, 2006.

Disclosure: M.B. Hoffmann, None; B. Lorenz, None; M. Preising, None; P.S. Seufert, None

The publication costs of this article were defrayed in part by page charge payment. This article must therefore be marked "advertisement" in accordance with 18 U.S.C. $\$ 1734$ solely to indicate this fact.

Corresponding author: Michael B. Hoffmann, Universitäts-Augenklinik, Visual Processing Lab, Leipziger Str. 44, 39120 Magdeburg, Germany; michael.hoffmann@medizin.uni-magdeburg.de. the retina is severely disrupted in albinism, where the line of decussation is shifted into the temporal retina, so that a great number of fibers from the temporal retina cross the midline and project contralaterally. ${ }^{1-7}$ As a consequence, the visual cortex receives abnormal input, which makes albinism a promising model to examine cortical self-organization in humans.

The extent to which the temporal retina is affected by the projection abnormality varies greatly among patients with albinism. $^{8-10}$ Therefore, a technique that samples the visual field for the abnormality with a high spatial resolution is necessary to describe the visual field topography of the projection abnormality. Such a technique would also help to identify abnormalities that are confined to small, circumscribed retinal areas that may be evident in carriers of albinism. Studies in cats have demonstrated small projection abnormalities in heterozygous carriers of albinism. ${ }^{11,12}$ Human carriers have so far been examined only with visual evoked potential (VEP) paradigms that did not allow for the differentiation of the projection pattern of distinct parts of the visual field. These studies failed to reveal any projection abnormalities, ${ }^{8,13,14}$ perhaps because of the local nature of the potential abnormalities. It is therefore of great interest to examine human carriers of albinism with a technique that samples the visual field for abnormal representations at a high spatial resolution.

VEPs are a valuable tool for identifying the misrouting of the optic nerves in human albinism. ${ }^{7,8,14-16}$ In albinism, each eye projects predominantly to its contralateral hemisphere. Monocular stimulation of the central visual field is therefore expected to elicit greater VEPs on the hemisphere contralateral to the stimulated eye than on the ipsilateral hemisphere. As a consequence, the interhemispheric VEP difference should invert its polarity for left compared with right eye stimulation in subjects with albinism, whereas it should not depend on the stimulated eye in control subjects. Apkarian et al. ${ }^{14}$ compared the polarity of the interhemispheric VEP differences obtained from occipital derivations for central stimulation of the left and right eyes. Indeed, they demonstrated the presence of inverted polarities in the subjects with albinism and its absence in the control subjects. This paradigm can be supplemented with a correlation analysis to simplify the approach and to enhance its objectivity: In albinism, the interhemispheric activation differences obtained for right and left eye stimulation are, due to the polarity inversion of the traces, likely to be negatively correlated. In contrast, in control subjects (i.e., in the absence of such a polarity inversion) the traces are likely to be positively correlated. ${ }^{10,16-18}$ This correlation approach therefore supports an objective analysis of even small signals.

Although conventional VEPs thus allow us to detect the projection abnormality typical for albinism, they do not allow us to screen the visual field at a high spatial resolution for the abnormality. This gap may be filled by multifocally recorded VEPs (mfVEPs). mfVEPs enable us to record cortical responses from a great number of distinct visual field locations within a short time interval and may therefore assist the identification of small projection abnormalities. ${ }^{19-22}$ The use of mfVEPs, however, is complicated by the convolution of the cortex, which 
results in a great intersubject variability of the responses and in false alarms in the detection of scotomas. ${ }^{20,23}$ Multielectrode recordings, ${ }^{24,25}$ interocular comparison of the responses, ${ }^{26,27}$ and refined analysis strategies ${ }^{22,24,28,29}$ have helped us to overcome these difficulties. Thus, mfVEPs open the possibility of identifying, with high spatial resolution, visual field locations that are affected by the projection abnormality that is typical of albinism.

We adapted previous conventional VEP approaches ${ }^{10,14,16}$ to identify projection abnormalities with mfVEPs. We correlated interhemispheric activation differences for left- and righteye stimulation at 60 different stimulus locations. Thus, we obtained visual field topographies of normal and abnormal representations. We pursued three goals with our study: (1) to demonstrate the projection abnormality in subjects with albinism; (2) to determine the specificity and sensitivity of the mfVEP approach in the detection of a projection abnormality; and (3) to investigate whether there are projection abnormalities in female carriers of ocular albinism (OA1).

\section{Materials ANd Methods}

\section{Subjects}

Five genetically confirmed female carriers of OA1 (aged 23-67 years; median 36) and 12 control subjects (aged 23-66 years; median 30), including five women age-matched with respect to the carriers (within \pm 3 years), participated in the study. Monocular visual acuity (Freiburg visual acuity test ${ }^{30}$ ), if necessary with refractive correction, was $\geq 0.9$. Eleven subjects with albinism (aged 22-54 years; median 37; nine men, two women; misrouting confirmed with the standard albino VEP paradigm $^{10,14}$ ) participated in the study; visual acuity ranged from 0.1 to 0.6 and horizontal nystagmus amplitude from $<0.4^{\circ}$ to $8.5^{\circ}$ (determined with the electro-oculogram). All subjects gave informed written consent before the study. The procedures complied with the tenets of the Declaration of Helsinki, ${ }^{31}$ and the protocol was approved by the ethics committee of the University of Freiburg, Germany.

\section{Stimulation}

A VEP system (VERIS 4.8; Electro-Diagnostic Imaging [EDI], San Mateo, CA) was used for stimulus delivery and electrophysiological recordings. Supported by a chin rest, subjects viewed the stimuli that were presented at a distance of $30 \mathrm{~cm}$ on a computer monitor driven with a frame rate of $75 \mathrm{~Hz}$. They were asked to fixate the center of a red cross that spanned the entire stimulus and thus served as a highly salient fixation target suitable for subjects with low visual acuity. The stimulus display, a circular dartboard pattern (see Fig. 4; diameter $45^{\circ}$; mean luminance $31 \mathrm{~cd} / \mathrm{m}^{2}$; contrast $95 \%$ ), was subdivided into individual fields, each comprising a checkerboard of $4 \times 4$ checks. The radial extent of the fields was scaled with eccentricity from $1.5^{\circ}$ in the center to $7^{\circ}$ in the periphery. The fields were stimulated independently with an m-sequence with $2^{15}-1$ elements. m-Sequences consist of a pseudorandom succession of 0 and 1 states. These two states were represented by two contrast-inverted checkerboard fields. Patternreversal responses can therefore be extracted as the first slice of the second-order kernel response.

\section{Electrophysiological Recordings}

Procedure. Recording sessions were conducted in a dimly lit room and lasted around 2 hours, including preparation and breaks. Two stimulus conditions, left- and right-eye stimulation, were presented in a balanced design (a-b-b-a scheme).

VEP Recordings. mfVEPs were recorded with six gold cup electrodes referenced to the inion. Electrodes were placed 4 and $8 \mathrm{~cm}$ left and right to the location $1 \mathrm{~cm}$ above the inion and at $\mathrm{P}_{3}$ and $\mathrm{P}_{4}{ }^{32}$ The EEG was amplified with a physiological amplifier (Toennies), band-pass filtered (low- and high-frequency cutoffs, 3 and $70 \mathrm{~Hz}$, respectively), and digitized at $1200 \mathrm{~Hz}$.

\section{Data Analysis}

Second-order kernels were extracted using VERIS 4.8 (EDI, Inc.). Spatial smoothing and artifact rejection features available in VERIS were not used. All subsequent analysis was performed with IGOR 5.0 (WaveMetrics, Inc., Lake Oswego, OR). The traces were digitally lowpass filtered with a high-frequency cutoff of $30 \mathrm{~Hz}$.

To assess signal presence we evaluated the signal-to-noise ratio (SNR), as described by Zhang et al. ${ }^{28}$ using a "mean noise-window SNR." First, the records from the two blocks for each stimulus were averaged. Then the SNR for each $i$ th sector (of the $n=60$ total sectors) of subject $j$ was defined as

$$
\mathrm{SNR}_{i j}=\mathrm{RMS}_{i j}(45-150 \mathrm{~ms}) /\left[\Sigma_{i} \operatorname{RMS}_{i j}(325-430 \mathrm{~ms}) / n\right]-1 .
$$

The denominator in (equation 1) is the average of the individual root mean squares (RMSs) of $n=60$ sectors in the noise window (325-430 $\mathrm{ms}$ after stimulus onset). An estimate of false-positive rates was obtained from the distribution of SNRs in the noise window for each $i$ th sector, $j$ th subject, $m$ th electrode pair, and $q$ th condition, according to Hood et al. ${ }^{24}$.

$$
\mathrm{SNR}_{i j m q}=\mathrm{RMS}_{i j m q}(325-430 \mathrm{~ms}) /\left[\Sigma_{i} \mathrm{RMS}_{i j m q}(325-430 \mathrm{~ms}) / n\right]-1 .
$$

Thus, we calculated $i \times j \times m \times q$ SNRs (i.e., 10,080 ratios; $i=60$ locations; $j=28$ subjects; $m=3$ electrode pairs; $q=2$ conditions [leftand right-eye stimulation]). An analysis of the distribution of these SNRs showed that SNRs $\geq 0.75$ are part of the noise distribution, with a probability of $<3 \%$. We therefore applied an SNR threshold of 0.75 to exclude "silent" visual field locations (i.e., without recordable signals) from our analyses. Thus, we included visual field locations with super-threshold responses (i.e., with recordable signals, which we will refer to as "responsive" locations). In our quantitative analyses we compared two stimulus conditions (i.e., left- and right-eye stimulation). Each stimulus location had to evoke superthreshold responses in at least one of the two conditions to enter the analysis (logical ORoperator). Thus, a bias of the results to one of these two conditions due to the thresholding procedure was avoided-for example, an AND operator would lead to an exclusion of stimulus locations that are suppressed below the SNR threshold in only one of the two stimulus conditions and would, as a consequence, cause an underestimation of possible interocular differences of the responses.

To assess the lateralization of the responses, we calculated the difference potentials for each of the three electrodes on one hemisphere and its corresponding electrode on the other hemisphere. We then selected for each visual field location the electrode pair with the greatest SNR during stimulation of either eye for further analysis. ${ }^{24}$ This procedure ensured that the same electrode pair was selected for leftand right-eye stimulation. Next, the difference VEPs obtained for each eye were correlated with each other to obtain Pearson's correlation coefficient ( $r$ ranging between -1 and 1 ). For this correlation the "signal time window" (45-150 ms) was used. The correlation allows for the distinction of normal and abnormal projections of the optic nerves. Positively correlated traces indicate that both eyes project to the same cortical regions, whereas negatively correlated traces indicate that both eyes project to opposite hemispheres. ${ }^{10,16}$ It should be noted that the correlation approach is a more objective approach than a single peak analysis and therefore allows one to deal with small signal amplitudes.

\section{Results}

\section{Qualitative Assessment of mfVEP Examples}

To assess the interhemispheric activation differences, the difference traces of the mfVEPs recorded at symmetrical elec- 
FIGURE 1. Interhemispheric mfVEP difference traces for a control (A), a carrier (B), and a subject with albinism (C) after left- (black trace) and right- (gray traces) eye stimulation. Traces are arranged according to the spatial layout of the visual field locations that evoked them. The traces from different eccentricities are arranged in an equidistant manner, whereas the actual stimulus layout is approximately $\mathrm{m}$-scaled. A magnification of the boxed traces is given in (D), together with an indication of the polarity of the peaks to indicate presence and absence of polarity reversals ( $\mathrm{p}$, positive peak; $n$, negative peak; abnormal succession of polarities is underlined for the subject with albinism). For the control and the carrier, the left- and right-eye responses are very similar (open arrows in $\mathbf{A}$ and $\mathbf{B}$; same polarities in D), whereas the polarities of the traces in the left compared with the right hemifield tended to be inverted (also depicted in $\mathbf{D}$ ). In the subject with albinism, these features (i.e., interocular similarity and inverted polarity of responses from opposing hemifields) only applied to the peripheral left and right visual fields (open arrow); for the responses along a vertical stripe in the central visual field, the polarity of the leftand right-eye responses was inverted (D, filled arrow in C) and there was an absence of a polarity reversal for the responses of a particular eye to left compared with right hemifield stimulation (D).
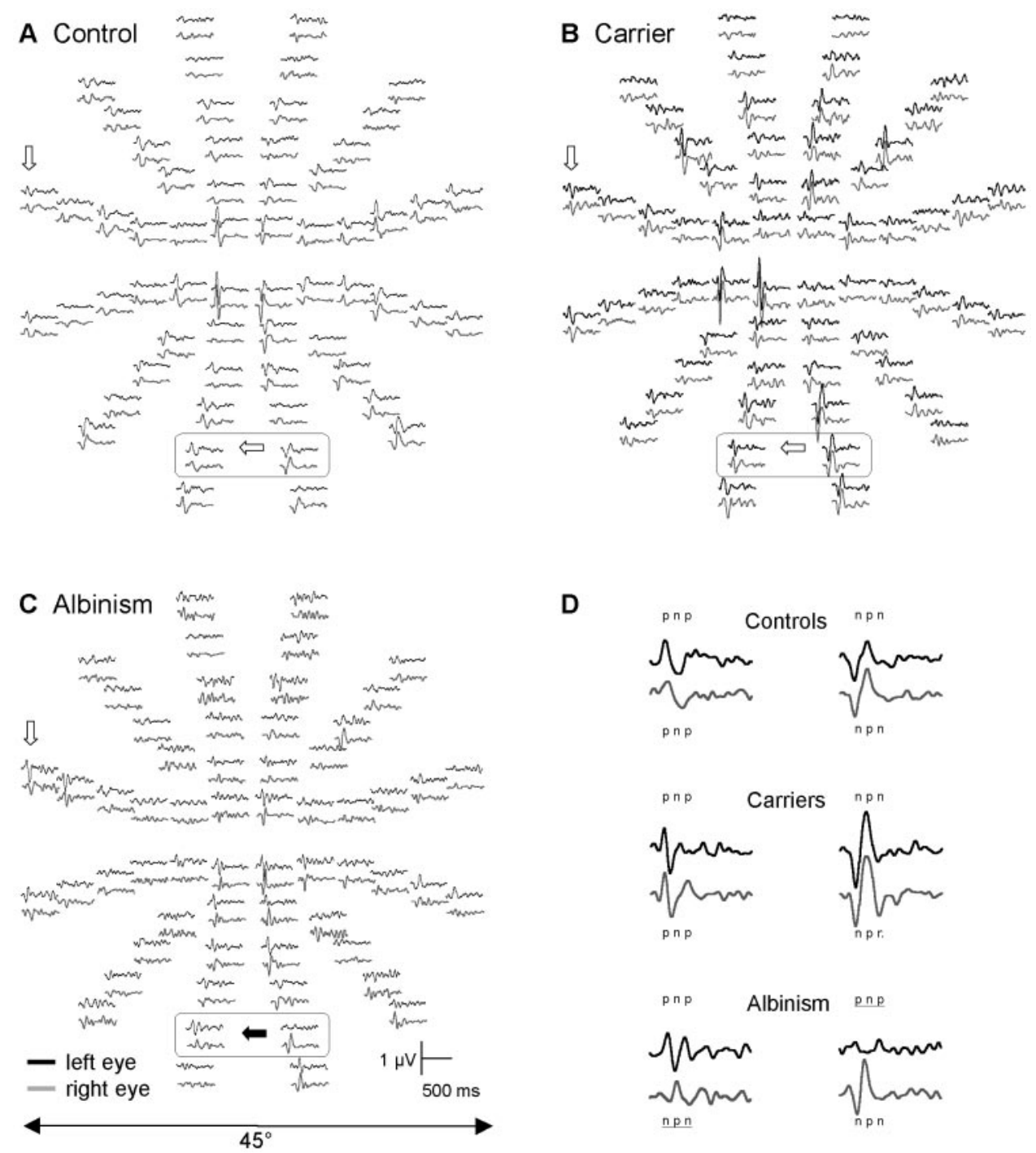

likely to create activity on opposite hemispheres. It therefore evokes interhemispheric activation differences of inverted polarity. Thus, a correlation of traces from opposing hemifields yielded mainly negative correlation coefficients, which will be detailed later. These features were also evident in the traces obtained from the female carrier (Figs. 1B, 1D). In the patient with albinism (Fig. 1C), there was a more differentiated picture. Responses to the peripheral left and right visual field displayed the characteristics already demonstrated in the control (i.e., interocular similarity and inverted polarity of responses from opposing hemifields). Responses along a vertical stripe in the central visual field, however, differed (Figs. 1C, 1D): (1) Response polarities after left- and right-eye stimulation were inverted (i.e., the responses are negatively correlated). (2) In a particular eye, there was no reversal of the polarity of the responses across the central vertical meridian. These features are indicative of the misrouting typical in subjects with albinism. Central responses to left- and right-eye stimulation are lateralized on right and left hemispheres, respectively, regardless of the hemifield stimulated.

\section{Efficacy of mfVEPs in Subjects with Albinism}

The overall efficacy of mfVEP recordings can be assessed from the number of responsive visual field locations. This number was equally high in control subjects and carriers of albinism 


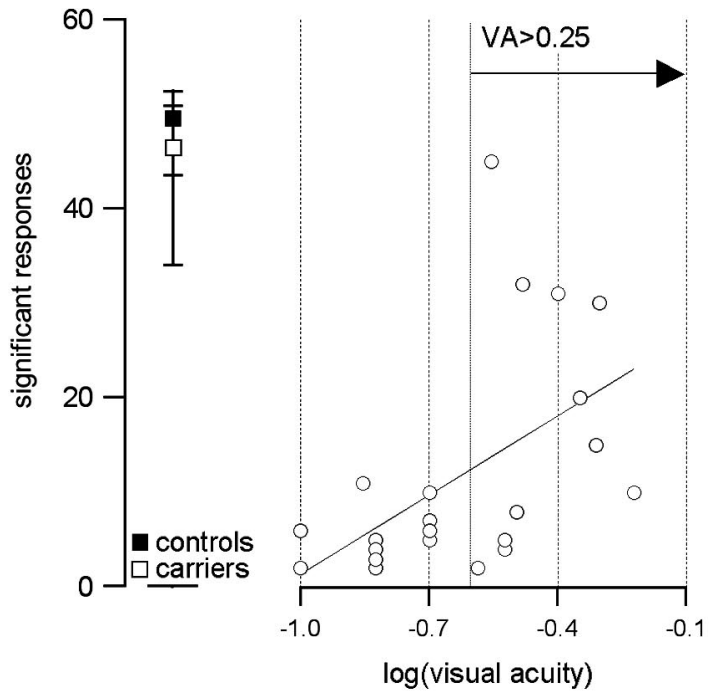

$\log$ (visual acuity)

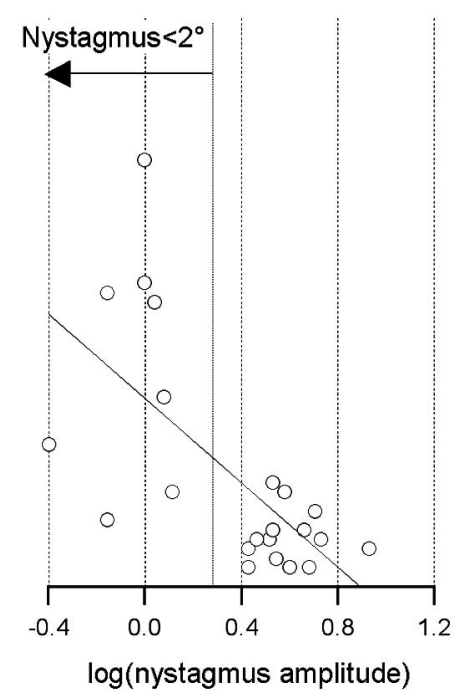

Figure 2. The number of responsive visual field locations. In the subjects with albinism, the number of responsive locations correlated with $\log$ (visual acuity) and $\log$ (nystagmus amplitude), as shown in the middle and at right $(n=22$ eyes). If visual acuity exceeded 0.25 and nystagmus was smaller than $2^{\circ}$ (solid lines, arrows), at least 10 of the 60 visual field locations stimulated were responsive. For comparison, the median \pm quartiles for the control subjects ( $n=24$ eyes) and the carriers $(n=10$ eyes $)$ are given at left (i.e., $83 \%$ and $78 \%$ of the total number of fields, respectively; Fig. 2, left). In albinism, a reduced number of visual field locations were responsive, which is related to both the amplitude of horizontal nystagmus and the subjects' visual acuity. The correlations with logarithmized nystagmus amplitude and visual acuity were significant at a level of $P=0.013$ and $P=$ 0.0009 , respectively. Consequently, comparatively high visual acuity and low nystagmus amplitude are necessary to evoke sizable responses in patients with albinism. A rough guideline can be derived from our data: If horizontal nystagmus amplitude was below $2^{\circ}$ and visual acuity above 0.25 , at least 10 visual field locations (i.e., $15 \%$ of the total number of fields) were responsive. In three of the patients tested, both eyes met these criteria, indicating the possibility of assessing the visual field topography of the projection abnormality in these patients. We will refer to these as patients with "sizable" responses.

\section{Visual Field Topography of the Interocular Correlation of the Difference mfVEPs}

As described earlier, the identification of abnormal projections can be assisted by the correlation of the interhemispheric mfVEP differences obtained for left- and right-eye stimulation: Positive interocular correlations indicate a normal, negative an abnormal projection of the optic nerves. In Figure 3, examples of the visual field topography of the correlations are given for a control subject, a carrier, and the three patients with albinism and sizable mfVEPs (see preceding section). In the control and the carrier, mfVEPs for all responsive visual field locations were positively correlated, which indicates that the visual field responses were lateralized on the same hemisphere for left- and right-eye stimulation. In the patients with albinism, response correlations are partly positive and partly negative, which corresponds to previous reports that demonstrated the projection abnormality only in a part of the visual field, mainly along a central vertical stripe. 9,10

\section{Quantitative Evaluation of the Correlation-Based Detection Procedure}

To assess the accuracy of the detection of the projection abnormality, we determined the specificity and the sensitivity of the detection procedure from the control data. This analysis was conducted for a threshold correlation coefficient of 0.0 as abnormal representations are expected to yield negative and normal positive correlation coefficients. ${ }^{10}$
Specificity. From the frequency distribution of the interocular correlation coefficients of the control subjects given in Figure $4 \mathrm{~A}$, it is evident that $3 \%$ of the correlation coefficients are negative, and $97 \%$ are positive. Consequently, for a threshold correlation coefficient of 0.0 , the probability of false alarms is $3 \%$, resulting in a specificity of the detection of an abnormal response lateralization of $97 \%$.

Sensitivity. In albinism, negative correlation coefficients indicate abnormal visual field representations on opposite hemispheres. The sensitivity (i.e., the hit rate), cannot be derived from subjects with albinism as it is not clear which locations are expected to be represented on opposite hemispheres and which are not. In control subjects the situation is different. Here, opposite hemifields are, due to the normal projection pattern, represented on opposite hemispheres. As a consequence, responses evoked by stimulation in opposite hemifields are expected to be negatively correlated. Thus, we correlated the responses of control subjects obtained after stimulation at visual field locations that are mirror symmetrical along the central vertical meridian. The resultant frequency distribution is shown in Figure 4B. It is evident that $75 \%$ of the correlation coefficients are negative. Consequently, for a threshold correlation coefficient of 0.0 , the sensitivity of the detection (i.e., the hit rate) is $75 \%$. Interhemispheric asymmetries of the cortical morphology and reduced amplitudes for some visual field locations may underlie this reduced sensitivity. An assessment of the dependence of the sensitivity on eccentricity in the control subjects did not reveal any significant relationships (repeated-measures ANOVA; comparison of three eccentricity bins comprising two adjacent annuli each). Neither did we find a pronounced reduction of the sensitivity along the vertical meridian (repeated-measures ANOVA; left plus right central vertical meridian versus other locations), which suggests that fixational eye movements are not a major cause of the sensitivity reduction.

Remarkably, proportions of negative correlation coefficients almost identical with those of the control subjects were obtained for the carriers of albinism for the interocular and the interhemifield correlation (i.e., $1 \%$ and $72 \%$, respectively; Figs. 4C, 4D). This similarity also holds, if the carriers are compared only to the five age- and sex-matched control subjects, which have $1 \%$ negative correlations for the interocular and $75 \%$ for the interhemifield correlation (data not shown). This is taken as evidence of an absence of a projection abnormality in the five carriers tested. The quantitative assessment of the correla- 

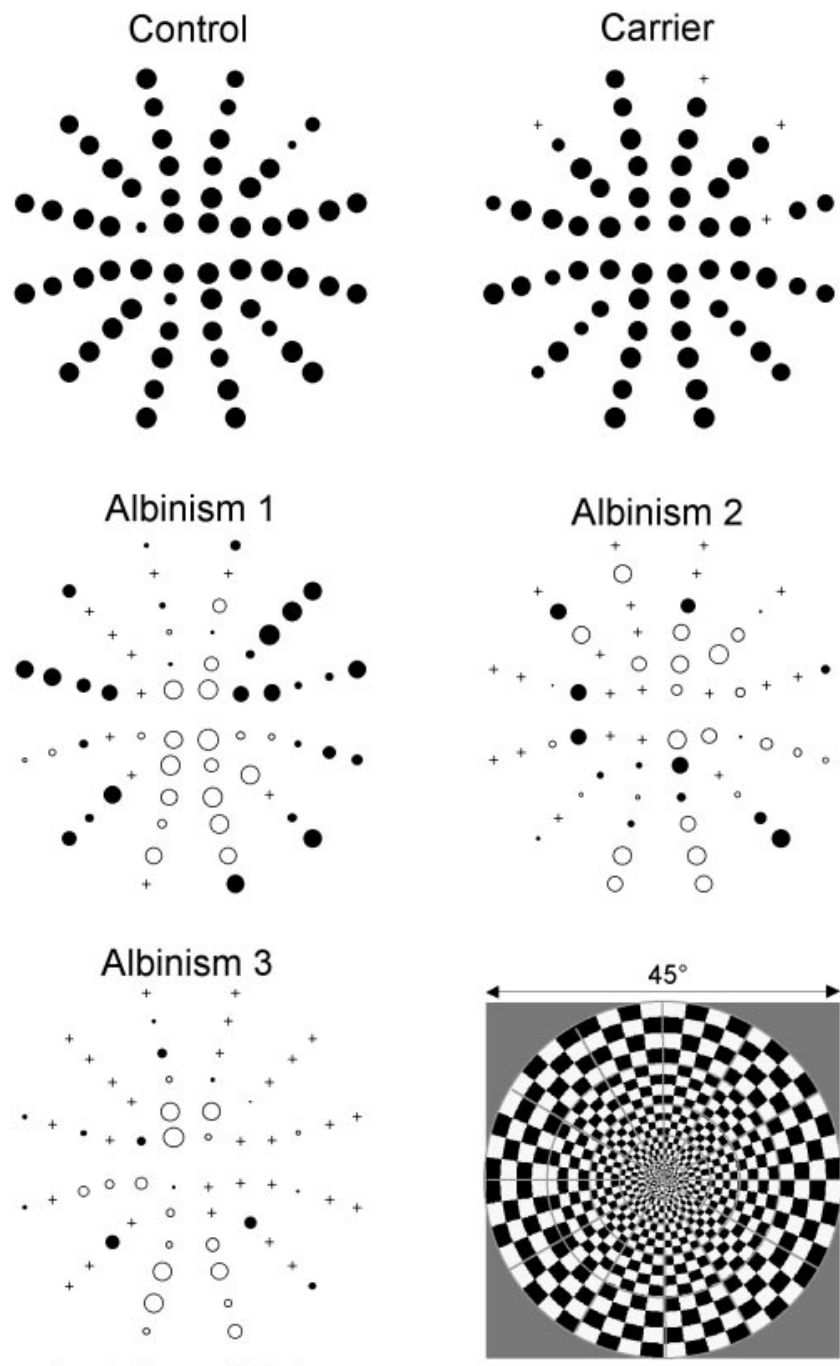

Correlation coefficient

$$
\begin{array}{ccccc}
-1.0 & -0.5 & 0 & 0.5 & 1.0 \\
0 & 0 & 0 & \bullet & 0
\end{array}
$$

FIGURE 3. Correlation of interhemispheric mfVEP differences in response to stimulation of the left and right eyes in a control subject, a carrier, and three subjects with albinism. The strength of the correlation is indicated by the diameter of the circles, the sign by its style: Solid symbols: a positive correlation (i.e., a normal projection pattern); open symbols: a negative correlation (i.e., an abnormal projection pattern); +, visual field locations with subthreshold responses. An abnormal projection pattern is evident only in the subjects with albinism, particularly in the central visual field. As a reference the stimulus pattern is included. Note that it is roughly m-scaled, whereas an equidistant depiction of the symbols is given for clarity.

tion coefficients is complemented by the frequency distribution obtained from the patients with albinism. In this case, it is due to the mixed abnormal and normal projection pattern of the optic nerves, expected that the correlation coefficients are partly positive and partly negative. Indeed, we obtained a flat distribution between -1 and 1 in these patients ( $49 \%$ of the correlation coefficients $<0.0 ; n=11$ ). However, as this also happens to resemble a noise distribution, it was of importance to evaluate whether the flat distribution might be due to noise. It must be noted that the intrusion of noise into the analysis is already minimized by the application of an SNR threshold, as detailed in the Methods section. If only a few visual field locations are responsive, however, residual noise leaking into the analysis has a potential to bias the analysis. We therefore applied a separate analysis including only the three patients with sizable responses (as defined above), to test whether a flat distribution is also obtained from a distribution, which is dominated by true responses. As a result, from this more conservative approach, we still obtained a flat distribution ( $55 \%$ of the correlations coefficients $<0.0 ; n=3$; see Fig. 4E), which is likely to reflect the heterogeneity of the projection of the optic nerve fibers in albinism: The part of the visual field locations that is affected by the projection abnormality yields negative values, the other part yields positive values, and the transition zone yields values around 0.0 .

For the distributions shown in Figures $4 \mathrm{~A}-\mathrm{E}$, the data from all individuals of the different subject groups were pooled. Finally, to assess the scatter of the individuals within a specific subject group, we determined the frequency of negative correlation coefficients for each subject. For a better comparability of control subjects and carriers we included for this analysis only age-related female control subjects. The population medians and upper and lower quartiles are depicted in Figure 4F. The results are similar to those shown in Figures 4A-E. We obtained $100 \%$ median specificity and $75 \%$ median sensitivity for the control subjects and similar proportions of correlation coefficients for the carriers (100\% and 67\%), which indicates a normal representation of the visual field in carriers. In contrast, a median of $43 \%$ of the visual field locations was misrepresented in albinism (for $n=11$ ). For the subset with sizable responses $(n=3)$ a median of $64 \%$ are misrepresented, as shown in Figure $4 \mathrm{~F}$.

\section{Discussion}

Correlation-based interocular comparison of interhemispheric mfVEP differences allowed us to detect visual field representations on opposite hemispheres with a specificity of $97 \%$ and a sensitivity of $75 \%$. In albinism, $55 \%$ of the visual field locations viewed by the left and right eyes were represented on opposing hemispheres. This is indicative of the misrouting of the optic nerves that is typical of albinism. Our results furthermore correspond to previous reports that demonstrated the representation abnormality of a part of the visual field, mainly along a central vertical stripe..$^{8-10}$ The detection of misrouting with VEPs may therefore benefit from the multifocal techniquehowever, as indicated by our data, only in subjects with negligible nystagmus and visual acuity $>0.25$.

\section{Misrouting in Carriers of Albinism?}

mfVEPs allow one to sample the visual field even for small representation abnormalities especially in subjects without nystagmus and with normal visual acuity. Carriers of albinism are therefore promising targets of this technique. In general, they have no signs of the visual dysfunction usually associated with albinism, but they may have some degree of misrouting. Animal studies report a projection abnormality, albeit reduced, in individuals who are heterozygous, normally pigmented carriers of albinism. ${ }^{11,12}$ In human carriers, this issue was previously investigated with the standard albino VEP paradigm, and no evidence of any misrouting was obtained, possibly because of the limited spatial resolution at which this approach samples the visual field. ${ }^{8,13,14}$ This absence of misrouting is even more surprising, as a subgroup of human carriers-namely, those of ocular albinism type 1 (OA1) - is likely to be affected by mild pigment deficits of fundus and iris, due to the $\mathrm{X}$-linked inheritance of OA1, which causes, via X-chromosomal inactivation, some cell lines to be normal and some to be abnormal. As a consequence, abnormal cell lines may cause an abnormal projection. However, even with the denser sampling of the visual 


\section{Correlation: Left \& right eye}
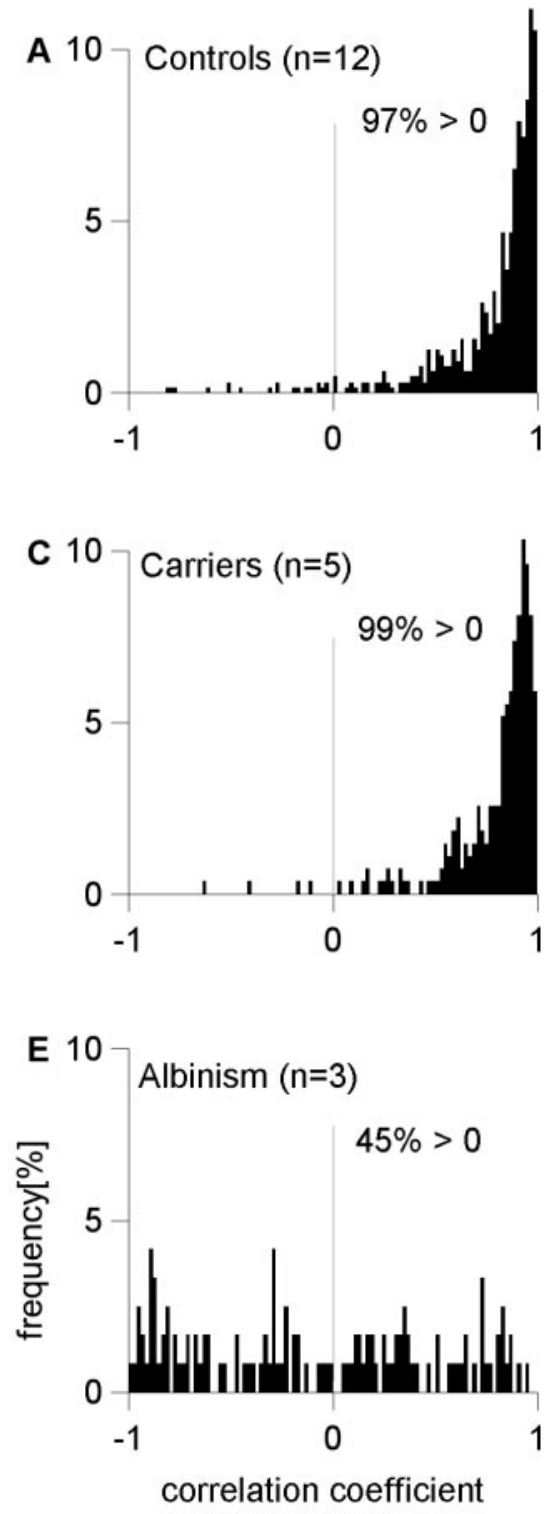

\section{Correlation: Left \& right hemifield}
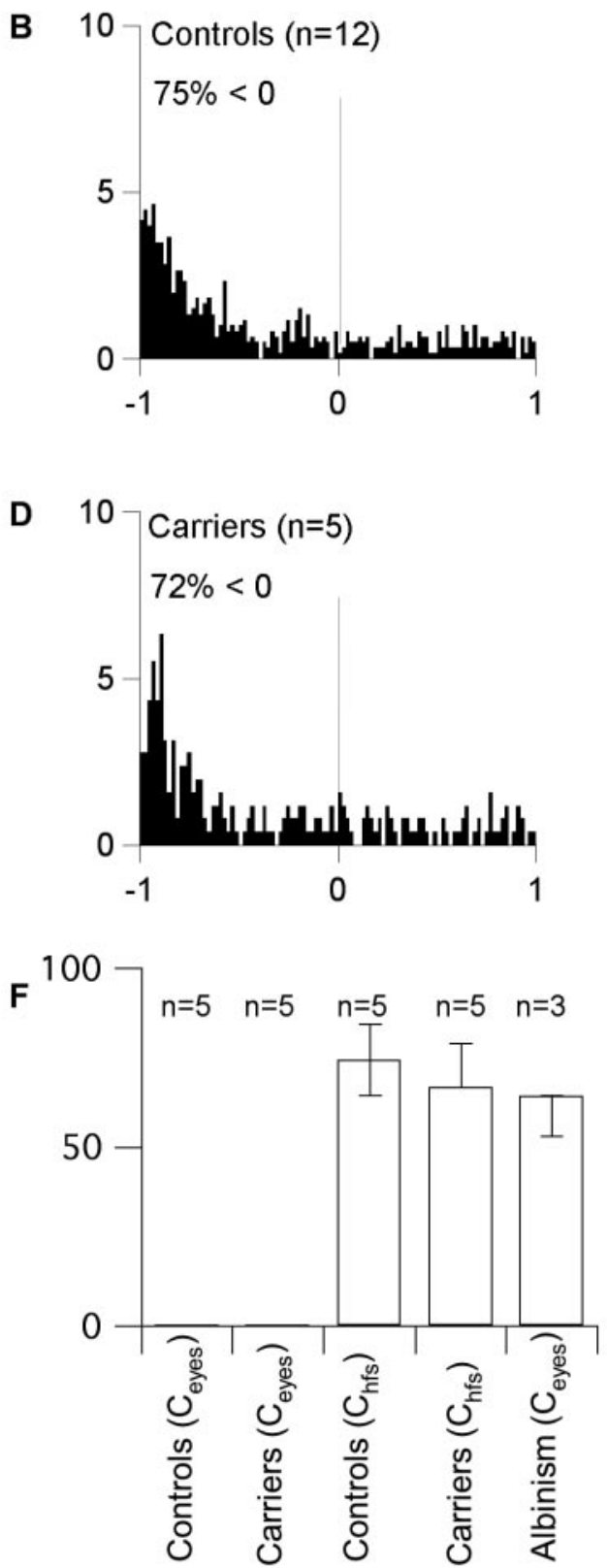

FiguRE 4. Frequency distribution of the correlation coefficients obtained in control subjects and carriers for stimulation of left and right eyes (A, C) and left and right hemifields (B, D). In the control subjects, the correlations of left- and right-eye responses (A) are, due to their representation on the same hemisphere, expected to yield positive coefficients. This measure is taken as an estimate of the specificity of the method: $97 \%$ of the visual field locations were correctly identified as normal. A similar distribution is evident in the carriers $(\mathbf{C} ; 99 \%>0)$. In contrast, only $45 \%$ of the visual field locations are positive in albinism, whereas $55 \%$ are negative (E), which indicates the abnormal representation of a proportion of the temporal retina. To estimate the sensitivity of the detection of visual field representations on opposing hemispheres, the left and right hemifield responses correlated in the control subjects (B). In fact, $75 \%$ of the visual field locations were correctly identified to be represented on opposing hemispheres. A similar distribution is evident in the carriers $(\mathbf{D} ; \mathbf{7 2} \%)$. (F) Frequencies of negative correlation coefficients are given as the median and upper and lower quartiles of the subjects of a particular subject group for the correlation of left and right eye $\left(\mathrm{C}_{\text {eyes }}\right)$ or hemifield $\left(\mathrm{C}_{\mathrm{hfs}}\right)$ responses (for better comparison only control subjects who were age- and sex-matched with the carriers were included in F). The results are similar to those shown in (A-E). field with mfVEPs, no evidence of misrouting was obtained in the carriers of OA1 tested.

Although these results may indicate the possibility of a dissociation of misrouting and local pigment deficits, the matter clearly deserves more attention. Because of the interindividual variability of the phenotypes of OA1 carriers, investigation of a larger sample of subjects is needed, and caution must be exerted in the interpretation of the findings in the present study, as only five carriers were investigated. It is also of importance to test carriers of the other types of albinism that follow the autosomal recessive inheritance-OCA1-as they would be the human equivalent of the individuals that tested positive for misrouting in the animal studies (i.e., normally pigmented heterozygotes for mutations on the tyrosinase locus). ${ }^{11,12}$ So far, we have shown that mfVEPs can be used to sample the visual field for projection abnormalities at a fine scale and that in none of the five carriers of OA1 studied was an indication of misrouting observed.

\section{Multifocal Stimulation Paradigm}

Might the detection procedure benefit from a stimulation mode other than pattern reversal? Pattern-reversal mfVEPs are a common tool for investigating representations in the visual cortex. They proved to be effective for the detection of visual field defects in several previous investigations, ${ }^{22,26,29,33}$ and the present study demonstrates their advantage for the detection of projection abnormalities, particularly in carriers, as these persons do not have fixation instabilities. An alternative stimulation mode, pattern-onset, also deserves attention. Although the investigation of subjects with pronounced nystagmus might benefit from stimulation in pattern-onset mode,${ }^{34-36}$ the investigation of the midperiphery (beyond $10^{\circ}$ ) 
of subjects without nystagmus (i.e., the carriers and control subjects of the present study) is likely to be impeded-in this region, pattern-onset mfVEPs are reduced compared with pattern-reversal mfVEPs. ${ }^{37}$ Further it should be noted, that traces of pattern-onset and pattern-reversal mfVEPs share a high degree of similarity (correlation coefficient $>0.6^{37}$ ) and are, as a consequence, likely to originate from the same generators. Both stimulus modes are therefore expected to be similarly effective for the detection of the projection pattern of the optic nerves with mfVEPs. This stands in contrast with conventional VEPs, in which lateralization differences of pattern-reversal and pattern-onset responses are evident ${ }^{38}$ that render pattern-onset preferable for the detection of response lateralizations with conventional VEPs. Detailed studies on the lateralization difference of pattern-onset and -reversal mfVEPs are needed to clarify this potential discrepancy of multifocal and conventional VEPs.

In the absence of oculomotor instabilities, mfVEPs allow us to detect the representation of visual field locations on opposite hemispheres with a high spatial resolution and are therefore a promising tool to test whether carriers of albinism are affected by small-scale projection abnormalities. In the five female carriers of OA1 investigated, we did not find evidence of such small-scale abnormalities.

\section{Acknowledgments}

The authors thank the subjects for their cooperation and Michael Bach for his kind permission to use his laboratory facilities.

\section{References}

1. Lund RC. Uncrossed visual pathways of hooded and albino rats. Science. 1965;149:1505-1507.

2. Creel DJ. Visual system anomaly associated with albinism in the cat. Nature. 1971;231:465-456.

3. Kaas JH, Guillery RW. The transfer of abnormal visual field representations from the dorsal lateral geniculate nucleus to the visual cortex in Siamese cats. Brain Res. 1973;59:61-95.

4. Guillery RW, Okoro AN, Witkop CJ Jr. Abnormal visual pathways in the brain of a human albino. Brain Res. 1975;96:373-377.

5. Hedera P, Lai S, Haacke EM, et al. Abnormal connectivity of the visual pathways in human albinos demonstrated by susceptibilitysensitized MRI. Neurology. 1994;44:1921-1926.

6. Schmitz B, Kasmann-Kellner B, Schafer T, et al. Monocular visual activation patterns in albinism as revealed by functional magnetic resonance imaging. Hum Brain Mapp. 2004;23:40 -52.

7. Morland AB, Hoffmann MB, Neveu M, Holder GE. Abnormal visual projection in a human albino studied with functional magnetic resonance imaging and visual evoked potentials. $J$ Neurol Neurosurg Psychiatry. 2002;72:523-526.

8. Creel D, Spekreijse H, Reits D. Evoked potentials in albinos: efficacy of pattern stimuli in detecting misrouted optic fibers. Electroencephalogr Clin Neurophysiol. 1981;52:595-603.

9. Hoffmann MB, Tolhurst DJ, Moore AT, Morland AB. Organization of the visual cortex in human albinism. J Neurosci. 2003;23:89218930.

10. Hoffmann MB, Lorenz B, Morland AB, Schmidtborn LC. Misrouting of the optic nerves in albinism: estimation of the extent with visual evoked potentials. Invest Opbthalmol Vis Sci. 2005;46:38923898.

11. Leventhal AG, Vitek DJ, Creel DJ. Abnormal visual pathways in normally pigmented cats that are heterozygous for albinism. Science. 1985;229:1395-1397.

12. Ault SJ, Leventhal AG, Vitek DJ, Creel DJ. Abnormal ipsilateral visual field representation in areas 17 and 18 of hypopigmented cats. J Comp Neurol. 1995;354:181-192.

13. Apkarian P. Electrodiagnosis in paediatric ophthalmogenetics. Int J Psychopbysiol. 1994;16:229-243.

14. Apkarian P, Reits D, Spekreijse H, van Dorp D. A decisive electrophysiological test for human albinism. Electroencephalogr Clin Neurophysiol. 1983;55:513-531.
15. Dorey SE, Neveu MM, Burton LC, Sloper JJ, Holder GE. The clinical features of albinism and their correlation with visual evoked potentials. Br J Ophthalmol. 2003;87:767-772.

16. Soong F, Levin AV, Westall CA. Comparison of techniques for detecting visually evoked potential asymmetry in albinism. J AAPOS. 2000;4:302-310.

17. Pott JW, Jansonius NM, Kooijman AC. Chiasmal coefficient of flash and pattern visual evoked potentials for detection of chiasmal misrouting in albinism. Doc Opbthalmol. 2003;106:137-143.

18. Tremblay F, De Becker I, Cheung C, LaRoche GR. Visual evoked potentials with crossed asymmetry in incomplete congenital stationary night blindness. Invest Opbthalmol Vis Sci. 1996;37:17831792.

19. Sutter EE. The fast m-transform: a fast computation of cross-correlations with binary m-sequences. SIAM J Comput. 1991;20:686694.

20. Baseler HA, Sutter EE, Klein SA, Carney T. The topography of visual evoked response properties across the visual field. Electroencepbalogr Clin Neurophysiol. 1994;90:65-81.

21. Klistorner AI, Graham SL, Grigg JR, Billson FA. Multifocal topographic visual evoked potential: improving objective detection of local visual field defects. Invest Ophthalmol Vis Sci. 1998;39:937950 .

22. Hood DC, Greenstein VC. Multifocal VEP and ganglion cell damage: applications and limitations for the study of glaucoma. Prog Retin Eye Res. 2003;22:201-251.

23. Hood DC, Zhang X. Multifocal ERG and VEP responses and visual fields: comparing disease-related changes. Doc Opbthalmol. 2000; 100:115-137.

24. Hood DC, Zhang X, Hong JE, Chen CS. Quantifying the benefits of additional channels of multifocal VEP recording. Doc Opbthalmol. 2002;104:303-320.

25. Klistorner AI, Graham SL. Objective perimetry in glaucoma. $O p h$ thalmology. 2000;107:2283-2299.

26. Graham SL, Klistorner AI, Grigg JR, Billson FA. Objective VEP perimetry in glaucoma: asymmetry analysis to identify early deficits. J Glaucoma. 2000;9:10-19.

27. Hood DC, Zhang X, Greenstein VC, Kangovi S, Odel JG. An interocular comparison of the multifocal VEP: a possible technique for detecting local damage to the optic nerve. Invest Ophthalmol Vis Sci. 2000;41:1580-1587.

28. Zhang X, Hood DC, Chen CS, Hong JE. A signal-to-noise analysis of multifocal VEP responses: an objective definition for poor records. Doc Ophthalmol. 2002;104:287-302.

29. Goldberg I, Graham SL, Klistorner AI. Multifocal objective perimetry in the detection of glaucomatous field loss. Am J Ophthalmol. 2002;133:29-39.

30. Bach M. The Freiburg Visual Acuity Test: automatic measurement of visual acuity. Optom Vis Sci. 1996;73:49-53.

31. World Medical Association. Declaration of Helsinki: ethical principles for medical research involving human subjects. JAMA. 2000; 284:3043-3045.

32. American Encephalographic Society. Guideline thirteen: guidelines for standard electrode position nomenclature. J Clin Neurophysiol. 1994;11:111-113.

33. Hood DC, Thienprasiddhi P, Greenstein VC, et al. Detecting early to mild glaucomatous damage: a comparison of the multifocal VEP and automated perimetry. Invest Opbthalmol Vis Sci. 2004;45: 492- 498 .

34. Hoffmann MB, Seufert PS. Simulated nystagmus reduces patternreversal more strongly than pattern-onset multifocal visual evoked potentials. Clin Neurophysiol. 2005;116:1723-1732.

35. Hoffmann MB, Seufert PS, Bach M. Simulated nystagmus suppresses pattern-reversal but not pattern-onset visual evoked potentials. Clin Neuropbysiol. 2004;115:2659-2665.

36. Saunders KJ, Brown G, McCulloch DL. Pattern-onset visual evoked potentials: more useful than reversal for patients with nystagmus. Doc Ophthalmol. 1998;94:265-274.

37. Hoffmann MB, Straube S, Bach B. Pattern-onset stimulation boosts central multifocal VEP responses. J Vis. 2003;3:432- 439.

38. Shawkat FS, Kriss A. A study of the effects of contrast change on pattern VEPS, and the transition between onset, reversal and offset modes of stimulation. Doc Opbthalmol. 2000;101:73-89. 